\title{
BIBLIOGRAPHY
}

1. M. R. Hestenes, An analogue of Green's theorem in the calculus of variations, Duke Math. J. vol. 8 (1941) pp. 300-311.

2. W. T. Reid, Green's lemma and related results, Amer. J. Math. vol. 63 (1941) pp. 563-574.

3. M. H. A. Newman, Elements of the topology of plane sets of points, Cambridge University Press, 1939.

HARVARD UNIVERSITY

\section{ON A CERTAIN TYPE OF NONLINEAR INTEGRAL EQUATIONS}

\section{MARK LOTKIN}

1. Introduction. The object of this paper is to prove that the nonlinear integral equation

$$
\phi(x)=\lambda\left[f(x)+\sum_{i=1}^{m} \int_{a}^{b} \cdots \int_{a}^{b} K_{i}\left(x, s_{1}, \cdots, s_{i}\right)\right.
$$

$$
\left.\cdot F_{i}\left(s_{1}, \cdots, s_{i}, \phi\left(s_{1}\right), \cdots, \phi\left(s_{i}\right)\right) d s_{1} \cdots d s_{i}\right]
$$

has at least one eigenvalue, provided the functionals

$$
\begin{aligned}
G_{i}(x, v)=\int_{a}^{b} \cdots \int_{a}^{b} K_{i}\left(x, s_{1}, \cdots, s_{i}\right) \\
\\
\qquad F_{i}\left(s_{1}, \cdots, s_{i}, v\left(s_{1}\right), \cdots, v\left(s_{i}\right)\right) d s_{1} \cdots d s_{i}
\end{aligned}
$$

are fully continuous, and the $F_{i}$ satisfy a certain linear integrodifferential equation. The solution of (1) is shown to be equivalent to that of a variational problem containing infinitely many parameters. The latter problem, however, can be solved easily by the method of Rayleigh-Ritz, which consists in approaching the solution of the variational problem by a sequence of variational problems containing only a finite number of parameters. The convergence of this procedure is assured by a convergence theorem of Friedrich Riesz.

2. Preparatory remarks. Let $I$ be the closed interval $a \leqq x \leqq b$, and $L^{2}$ the class of all functions having Lebesgue integrable squares on $I$ with a norm not larger than $N^{2}$. Let, further, $\left\{v_{n}(x)\right\}(n=1,2,3, \cdots)$

Received by the editors May 18, 1944. 
be a set of functions in $L^{2}$ and $\bar{v}(x)$ a function such that ${ }^{1}$

$$
\lim _{n \rightarrow \infty} \int v_{n}(x) w(x) d x=\int \bar{v}(x) w(x) d x
$$

for any arbitrary $w$ of integrable square on $I$ or, what is equivalent, for any arbitrary $w \in L^{2}$; that is, we assume the weak convergence of $\left\{v_{n}\right\}$, in the following denoted by $W-\lim _{n \rightarrow \infty} v_{n}=\bar{v}$. We next show that $\bar{v} \in L^{2}$. Since the right-hand side of (3) is assumed to exist for every $w \in L^{2}$, it follows by a known result (see, for example, KaczmarzSteinhaus, Theorie der Orthogonalreihen) that $\bar{v}$ is of integrable square over $I$. Hence, if $w=\bar{v}$, (3) becomes

$$
\int \bar{v}^{2}(x) d x=\lim _{n \rightarrow \infty} \int v_{n}(x) \bar{v}(x) d x,
$$

and since by Schwarz's inequality

$$
\left[\int v_{n}(x) \bar{v}(x) d x\right]^{2} \leqq N^{2}\left[\int \bar{v}^{2}(x) d x\right],
$$

we obtain, as claimed,

$$
\int \bar{v}^{2}(x) d x \leqq N^{2} .
$$

We now assume that the functional $G_{i}(x, v)$ be fully continuous, that is, that

$$
\lim _{n \rightarrow \infty} G_{i}\left(x_{n}, v_{n}\right)=G_{i}(\bar{x}, \bar{v}), \quad i=1,2, \cdots, m,
$$

for any $\left\{x_{n}\right\} \in I$ and $v_{n} \in L^{2}$ for which $\lim _{n \rightarrow \infty} x_{n}=x$ and $W-\lim _{n \rightarrow \infty} v_{n}=\bar{v}$.

The introduction of a closed orthonormal system of functions $\left\{w_{\nu}(x)\right\} \in L^{2}$ associates with each $v_{n}$ an infinite sequence of numbers

such that

$$
c_{n \nu}=\int v_{n}(x) w_{\nu}(x) d x, \quad \nu=1,2, \cdots,
$$

$$
\sum_{\nu=1}^{\infty} c_{n \nu}^{2}=\int v_{n}^{2}(x) d x \leqq N^{2}
$$

for every $n \geqq 1$. The class $L^{2}$ of functions $v$ then corresponds to a class $\mathfrak{S}^{2}$ of vectors $\mathfrak{b} \equiv\left(c_{1}, c_{2}, \cdots\right)$ with $c_{\nu}=\int v(x) w_{\nu}(x) d x$. The transition

1 All integrations are to be extended over the interval $I$. 
from $L^{2}$ to $\mathfrak{S}^{2}$ implies the substitution in $G_{i}\left(x_{n}, v_{n}\right)$ of $v_{n}(x)$ by its equivalent $\mathfrak{b}(x) \sim \sum_{\nu=1}^{\infty} c_{n \nu} w_{\nu}(x)$, and (5) now changes to

$$
\lim _{n \rightarrow \infty} G_{i}\left(x_{n}, v_{n}\right)=G_{i}(\bar{x}, \bar{v}) \equiv P_{i}\left(\bar{x}, \bar{c}_{1}, \bar{c}_{2}, \cdots\right)
$$

for any $\left\{x_{n}\right\} \in I$ and $\left\{v_{n}\right\} \in \mathscr{S}^{2}$ for which $\lim _{n \rightarrow \infty} x_{n}=\bar{x}$ and $\lim _{n \rightarrow \infty} c_{n \nu}$ $=\bar{c}_{\nu}, \nu=1,2, \cdots$. From the full continuity of the $G_{i}$ thus follows the full continuity (Vollstetigkeit) ${ }^{2}$ of the $P_{i}$. It is just as easily seen that the converse also holds true.

3. Construction of a solution of the integral equation. In the $G_{i}$ we now admit as arguments $v_{n}$ only aggregates of the form $v_{n}(x)$ $=\sum_{\nu=1}^{n} c_{n \nu} w_{\nu}(x)$ with $\sum_{\nu=1}^{n} c_{n \nu}^{2}=N^{2}$ for every fixed $n$. The functional

$$
J\left(v_{n}\right)=2\left[\int f(x) v_{n}(x) d x+\sum_{i=1}^{m} e_{i} \int G_{i}\left(x, v_{n}\right) v_{n}(x) d x\right]
$$

- here the $e_{i}$ denote finite numbers to be determined later-is now a continuous function of the $c_{n \nu}$ and so has at least one minimum. Let $c_{n \nu}=a_{n \nu}(\nu=1,2, \cdots, n)$ be the minimal coordinates:

(7) $\quad \min J\left(v_{n}\right)=d_{n}=J\left(\phi_{n}\right), \quad \phi_{n}(x)=\sum_{\nu=1}^{n} a_{n \nu} w_{\nu}(x), \quad \sum_{\nu=1}^{n} a_{n \nu}^{2}=N^{2}$.

As a consequence of (7) we have ${ }^{8}$

(8) $\frac{\partial}{\partial c_{n \nu}}\left[J\left(v_{n}\right)+\frac{1}{\lambda_{n}}\left(N^{2}-\sum_{\mu=1}^{n} c_{n \mu}^{2}\right)\right]_{c_{n \nu}-a_{n \nu}}=0, \quad \nu=1,2, \cdots, n$.

However,

$$
\begin{aligned}
\frac{\partial J\left(v_{n}\right)}{\partial c_{n \nu}}=2\left[\int f w_{\nu} d x+\sum_{i} e_{i} \iint\right. & \ldots \\
& \left.\int K_{i}\left(v_{n} \frac{\partial F_{i}}{\partial c_{n \nu}}+w_{\nu} F_{i}\right) d x d s_{1} \cdots d s_{i}\right] .
\end{aligned}
$$

We must now make the following assumption: The $F_{i}$ satisfy the linear integrodifferential equations

$$
\iint \cdots \int K_{i}\left[e_{i} v_{n} \frac{\partial F_{i}}{\partial c_{n \nu}}-\left(1-e_{i}\right) w_{\nu} F_{i}\right] d x d s_{1} \cdots d s_{i}=0
$$

${ }^{2}$ See D. Hilbert, Grundzilge einer allgemeinen Theorie der linearen Integralgleichungen, 1912, p. 177. A fully continuous function $P\left(x, c_{1}, c_{2}, \cdots\right)$, where $x \in I$ and $\sum_{r=1}^{\infty} c_{r} \leqq N^{2}$, is bounded.

${ }^{8}$ In (8), $1 / \lambda_{n}$ denotes Lagrange's multiplier for the extremum problem under consideration. As will be shown subsequently, $\lambda_{n} \neq 0$ for every $n$. 
for all arguments $v_{n}=\sum_{(\nu)} c_{n \nu} w_{\nu}$, identically in the $c_{n \nu}$.

In this case we obtain

$$
\frac{\partial J\left(v_{n}\right)}{\partial c_{n \nu}}=2\left[\int\left(f+\sum_{i} \int \cdots \int K_{i} F_{i} d s_{1} \cdots d s_{i}\right) w_{\nu} d x\right],
$$

and (8) leads to

$$
a_{n \nu}=\lambda_{n} \int G\left(x, \phi_{n}\right) w_{\nu} d x, \quad \nu=1,2, \cdots, n,
$$

with

$$
\begin{aligned}
G\left(x, \phi_{n}\right)=f(x)+\sum_{i} \int & \cdots \int K_{i}\left(x, s_{1}, \cdots, s_{i}\right) \\
& \cdot F_{i}\left(s_{1}, \cdots, s_{i}, \phi_{n}\left(s_{1}\right), \cdots, \phi_{n}\left(s_{i}\right)\right) d s_{1} \cdots d s_{i} .
\end{aligned}
$$

On account of $a_{n \nu}=\int \phi_{n} w_{\nu} d x$ the relations (10) may be written as

$$
\int\left(\phi_{n}-\lambda_{n} G\left(x, \phi_{n}\right)\right) w_{\nu} d x=0 \quad \text { for } \nu=1,2, \cdots, n .
$$

Equations (10) show that the $\left|\lambda_{n}\right|$ have a common positive lower bound: multiplication of (10) by $a_{n \nu}$ and summation for $\nu=1, \cdots, n$ result in

$$
N^{2}=\lambda_{n} \int G\left(x, \phi_{n}\right) \phi_{n} d x .
$$

But since $G\left(x, \phi_{n}\right)$ is a fully continuous function of the $a_{n v}$ for $x \in I$ and $\phi_{n} \in H^{2}$ it is bounded: there exists a $\delta>0$ such that

Therefore

$$
\left|G\left(x, \phi_{n}\right)\right| \leqq N /(b-a)^{1 / 2} \delta \text { for every } n .
$$

$$
\left|\int G\left(x, \phi_{n}\right) \phi_{n} d x\right| \leqq\left[\int G^{2}\left(x, \phi_{n}\right) d x\right]^{1 / 2}\left[\int \phi_{n}^{2} d x\right]^{1 / 2} \leqq N^{2} / \delta
$$

whence $\left|\lambda_{n}\right| \geqq \delta>0$ for every $n$.

Now the $\int \phi_{n}^{2} d x$ all have the same value $N^{2}$. This property of the sequence $\left\{\phi_{n}\right\}$ guarantees the existence of a $\phi(x)$-defined almost everywhere in $I$ and possessing a Lebesgue integrable square-which is the $W$-lim of a suitably chosen subsequence $\left\{\phi_{n}\right\}$ of $\left\{\phi_{n}\right\}, 4$

' Friedrich Riesz, Untersuchungen uber Systeme integrierbarer Funktionen, Math. Ann. vol. $69(1910)$ p. 467 . The sequence $\{\bar{n}\}$ is determined by Hilbert's diagonal method (see, for example, Hellinger-Toeplitz, Encyklopädie der mathematischen Wissenschaften vol. II C 13, p. 1405). 


$$
W-\lim _{n \rightarrow \infty} \phi_{\bar{n}}(x)=\bar{\phi}(x)
$$

because the system $\left\{w_{\nu}\right\}$ is closed $\Phi$ is determined uniquely almost everywhere in $I$. On account of $(4), \int \Phi^{2}(x) d x \leqq N^{2}$.

We are now going to show that

$$
\lim _{\bar{n} \rightarrow \infty} \int G\left(x, \phi_{\bar{n}}\right) \phi_{\bar{n}} d x=\int G(x, \bar{\phi}) \bar{\phi} d x .
$$

Since

$$
\begin{aligned}
\left|\int G(x, \bar{\phi}) \bar{\phi} d x-\int G\left(x, \phi_{\bar{n}}\right) \phi_{\bar{n}} d x\right| \leqq & \int G(x, \bar{\phi})\left(\bar{\phi}-\phi_{\bar{n}}\right) d x \mid \\
& +\left|\int\left(G(x, \bar{\phi})-G\left(x, \phi_{\bar{n}}\right)\right)_{\bar{n}} d x\right|,
\end{aligned}
$$

and the first expression on the right hand side-by (13)-may be made as small as desired by taking $\bar{n}$ sufficiently large, only the second term remains to be considered. Now

$$
\left|\int\left(G(x, \bar{\phi})-G\left(x, \phi_{\bar{n}}\right)\right) \phi_{\bar{n}} d x\right| \leqq N\left[\int\left(G(x, \bar{\phi})-G\left(x, \phi_{\bar{n}}\right)\right)^{2} d x\right]^{1 / 2},
$$

and so (14) will be proved if we can show that $\lim _{n \rightarrow \infty} \int(G(x, \bar{\phi})$ $\left.-G\left(x, \phi_{n}\right)\right)^{2} d x=0$. This, however, follows immediately from the convergence theorem of Lebesgue. ${ }^{5}$ The sequence $L_{n} \equiv\left(G(x, \bar{\phi})-G\left(x, \phi_{n}\right)\right)^{2}$ obviously satisfies the conditions of that theorem: (a) $L_{n}$ is Lebesgue integrable; (b) Since $|G(x, v)| \leqq N /(b-a)^{1 / 2} \delta,\left|L_{n}\right| \leqq 4 N^{2} /(b-a) \delta^{2}$ for every $\bar{n}$; (c) Because of the full continuity of $G(x, v), \lim _{n \rightarrow \infty}(G(x, \bar{\phi})$ $\left.-G\left(x, \phi_{n}\right)\right)=0$. Therefore $\bar{L}=0$, which proves (14).

We must now distinguish between these two cases:

I. There exists a $\delta^{\prime}>0$ such that $\left|\int G(x, \bar{\phi}) \Phi d x\right| \geqq N^{2} / \delta^{\prime}$;

II. $\int G(x, \Phi) \Phi d x=0$.

CASE I. By (12), $\lambda_{n}=N^{2} / \int G\left(x, \phi_{n}\right) \phi_{n} d x$, so that by (14)

$$
\lim _{\bar{n} \rightarrow \infty} \lambda_{\bar{n}}=\bar{\lambda}=\frac{N^{2}}{\int G(x, \bar{\phi}) \bar{\phi} d x}
$$

exists; because of $I,|\bar{\lambda}| \leqq \delta^{\prime}$.

- If a sequence of Lebesgue integrable functions $L_{n}(x)$ possessing a common bound has a limit function $\bar{L}(x)$, then $\bar{L}$, too, is Lebesgue integrable and $\lim _{n \rightarrow \infty} \int L_{n}(x) d x$ $=\int \mathbb{L}(x) d x$. 
If we now apply equations (11) to indices $\pi$ only and then take the $\lim _{n \rightarrow \infty}$ we obtain

$$
\int(\bar{\phi}(x)-\bar{r}(x, \bar{\phi})) w_{\nu}(x) d x=0 \quad \text { for } \nu=1,2, \ldots .
$$

Since the system of the $\left\{w_{\nu}\right\}$ is closed we may deduce $\bar{\phi}-\bar{\lambda} G(x, \bar{\phi})=0$, that is,

$$
\begin{aligned}
\Phi(x)=\bar{\lambda}\left[f(x)+\sum_{i=1}^{m} \int \cdots\right. & \int K_{i}\left(x, s_{1}, \cdots, s_{i}\right) \\
& \left.\cdot F_{i}\left(s_{1}, \cdots, s_{i}, \Phi\left(s_{1}\right), \cdots, \Phi\left(s_{i}\right)\right) d s_{1} \cdots d s_{i}\right]
\end{aligned}
$$

almost everywhere in $I$. We have thus obtained a solution $\bar{\phi}(x)$ of (1) belonging to the finite eigenvalue $\bar{\lambda}$.

The previously derived relationship $\int \phi^{2}(x) d x \leqq N^{2}$ may now be improved: replacing $G(x, \bar{\phi})$ in (15) by its equal $(1 / \bar{\lambda}) \bar{\phi}$ leads to $\int \phi^{2}(x) d x=N^{2}$.

CASE II. We write equations (10) for indices $\bar{n}$ only:

$$
\int G\left(x, \phi_{n}\right) w_{\nu} d x=\frac{1}{\lambda \bar{n}} a_{\tilde{n} v}, \quad \nu=1,2, \cdots, \bar{n},
$$

and increasing $\bar{n}$ beyond any bound we obtain, since $\lim _{n \rightarrow \infty} \lambda_{n}=\infty$ and $\left|a_{n \nu}\right| \leqq N$,

$$
\int G(x, \phi) w_{\nu} d x=0, \quad \nu=1,2, \cdots .
$$

In this case $\phi(x)$ may be considered a solution of (1) belonging to $\lambda=\infty$.

4. The variational problem. We see, then, that $\phi$ is always a solution of (1). This function possesses another important property: If $\overline{\mathscr{S}}^{2}$ denotes the class of all $\mathfrak{b}(x) \sim \sum_{(v)} c_{\nu} w_{\nu}(x)$ with $\sum_{(\nu)} c_{\nu}^{2}=N^{2}$, and

$$
\mathfrak{b}(x) \sim \sum_{\nu=1}^{\infty} \bar{a}_{\nu} w_{\nu}(x) \quad \text { with } \quad \bar{a}_{\nu}=\int \Phi(x) w_{\nu}(x) d x,
$$

then $\mathfrak{b}$ minimizes $J(\mathfrak{b})$.

To prove this we notice first that $J\left(\mathfrak{b}_{n}\right)$ results from $J\left(\mathfrak{b}_{n+1}\right)$ if we put $c_{n+1}=0$. Let $d_{n}$ be the minimum of $J\left(\mathfrak{b}_{n}\right)$ in $\overline{\mathfrak{W}}^{2}$. Then obviously $d_{n} \geqq d_{n+1}$. Let, further, $d$ be the minimum of $J(\mathfrak{v})$ for $\mathfrak{v} \in \overline{\mathbb{S}}^{2}$; then $d_{n} \geqq d$ for every $n$. Therefore, if $\bar{d}=\lim _{n \rightarrow \infty} d_{n}=J(\mathfrak{b})$, we get $\bar{d} \geqq d$ or $\bar{d}=d+\eta$ with $\eta \geqq 0$. We shall show that $\eta=0$. 
Since $d$ is the lower bound of $J(\mathfrak{b})$ in $\overline{\mathfrak{W}}^{2}$ there exists a $\mathfrak{p}(x)$ $\sim \sum_{(\nu)} p_{\nu} w_{\nu}(x)$ in $\overline{\mathfrak{S}}^{2}$ so that $J(\mathfrak{p})=\bar{d}-\theta \eta$ with $0<\theta \leqq 1$. If, now, $\epsilon>0$ be chosen as small as desired, there is, because of the full continuity of $J(\mathfrak{b})$, a $\delta>0$ and an index $r$ such that $|J(\mathfrak{b})-J(\mathfrak{p})|<\epsilon$ for every $\mathfrak{v} \in \overline{\mathfrak{S}}^{2}$, so long as $\left|c_{\nu}-p_{\nu}\right|<\delta$ for $\nu=1,2, \cdots, r$. We take $\epsilon=\theta \eta$ and choose $r^{\prime} \geqq r$ large enough to have $\sum_{\nu=1}^{\gamma^{\prime}} p_{\nu}^{2}=N^{\prime 2}>N^{2} \cdot N^{2} /(N+\delta)^{2}$. Then the vector $\mathfrak{\mathfrak { p }}(x)=\sum_{\nu=1}^{r^{\prime}} \bar{p}_{\nu} w_{\nu}(x)$ with $\bar{p}_{\nu}=\left(N / N^{\prime}\right) p_{\nu}$ belongs to $\overline{\mathfrak{W}}^{2}$, and since $N^{\prime}>N^{2} /(N+\delta)$,

$$
\left|p_{\nu}-p_{\nu}\right|=\left|p_{\nu}\right| \cdot\left(N / N^{\prime}-1\right) \leqq N\left(N / N^{\prime}-1\right)<\delta
$$

for $\nu=1,2, \cdots, r^{\prime}$. We may, therefore, conclude that $|J(\mathfrak{p})-J(\mathfrak{p})|$ $<\theta \eta$ or $J(\mathfrak{p})<J(\mathfrak{p})+\theta \eta=\bar{d}$. But $d_{r^{\prime}} \leqq J(\mathfrak{p})$, and so $d_{r^{\prime}}<\bar{d}$.

By now choosing $\bar{n}, \bar{n} \geqq r^{\prime}$, such that $d_{n} \leqq d_{r^{\prime}}$ we get $d_{n} \leqq \bar{d}$, a relation which contradicts the fact that the sequence $d_{n}$ converges to $\bar{d}$ from above. Thus we see that $\eta=0$ or $\bar{d}=J(\mathfrak{b})$.

5. Solution of the integrodifferential equation. It is easy to verify that equations (9) are fulfilled if we put $e_{i}=1 /(i+1), K_{i}$ continuous and

$$
\begin{gathered}
K_{i}\left(x, s_{1}, \cdots, s_{k}, \cdots, s_{i}\right)=K_{i}\left(s_{k}, s_{1}, \cdots,\right. \\
\quad \begin{array}{r}
\left.x, \cdots, s_{i}\right), \\
k=1,2, \cdots, i,
\end{array} \\
F_{i}\left(s_{1}, \cdots, s_{i}, u_{1}, \cdots, u_{i}\right)=a_{i} u_{1} \cdots u_{i}
\end{gathered}
$$

for $i=1,2, \cdots, m$.

It remains to be shown that functionals of the type

$$
Q(x, v)=\int \cdots \int K\left(x, s_{1}, \cdots, s_{i}\right) v\left(s_{1}\right) \cdots v\left(s_{i}\right) d s_{1} \cdots d s_{i}
$$

are fully continuous for $x \in I$ and $v \in L^{2}$. Let us, therefore, assume that $\left\{x_{n}\right\} \in I,\left\{v_{n}\right\} \in L^{2}, \lim _{n \rightarrow \infty} x_{n}=\bar{x}$, and $W-\lim _{n \rightarrow \infty} v_{n}=\bar{v}$. Then

$$
\begin{aligned}
\left|Q(\bar{x}, \bar{v})-Q\left(x_{n}, v_{n}\right)\right| \leqq & \left|Q(\bar{x}, \bar{v})-Q\left(\bar{x}, v_{n}\right)\right|+\left|Q\left(\bar{x}, v_{n}\right)-Q\left(x_{n}, v_{n}\right)\right| \\
= & \mid \int \cdots \int K\left(\bar{x}, s_{1}, \cdots, s_{i}\right)\left[\bar{v}\left(s_{1}\right) \cdots \bar{v}\left(s_{i}\right)\right. \\
& \left.-v_{n}\left(s_{1}\right) \cdots v_{n}\left(s_{i}\right)\right] d s_{1} \cdots d s_{i} \mid \\
+ & \\
& \left.\quad-K\left(x_{n}, s_{1}, \cdots, s_{i}\right)\right] v_{n}\left(s_{1}\right) \cdots v_{n}\left(s_{i}\right) d s_{1} \cdots d s_{i} \mid .
\end{aligned}
$$


Because of the continuity of $K$ the second term on the right-hand side may be made arbitrarily small by choosing $n$ sufficiently large. In order to show that the same applies also to the first term we continue as follows:

$$
\begin{aligned}
& \mid \int \cdots \int K\left[\bar{v}\left(s_{1}\right) \cdots \bar{v}\left(s_{i}\right)-v_{n}\left(s_{1}\right) \cdots v_{n}\left(s_{i}\right)\right] d s_{1} \cdots d s_{i} \mid \\
&= \mid \int \cdots \int K \sum_{k=1}^{i} \bar{v}\left(s_{1}\right) \cdots \bar{v}\left(s_{k-1}\right)\left[\bar{v}\left(s_{k}\right)-v_{n}\left(s_{k}\right)\right] \\
& \cdot v_{n}\left(s_{k+1}\right) \cdots v_{n}\left(s_{i}\right) d s_{1} \cdots d s_{i} \mid \\
& \leqq \\
& \sum_{k=1}^{i} \mid \int \cdots \int\left(\int K\left[\bar{v}\left(s_{k}\right)-v_{n}\left(s_{k}\right)\right] d s_{k}\right) \\
& \leqq \sum_{k=1}^{i}\left\{\int \cdots \int\left(\int K\left[\bar{v}\left(s_{k}\right)-v_{n}\left(s_{k}\right)\right] d s_{k}\right)^{2} \cdots \bar{v}\left(s_{k-1}\right) v_{n}\left(s_{k+1}\right) \cdots v_{n}\left(s_{i}\right) d s_{1} \cdots d s_{i} \mid\right. \\
&\left.\cdot d s_{1} \cdots d s_{k-1} d s_{k+1} \cdots d s_{i}\right\}^{1 / 2} \cdot N^{i-1} .
\end{aligned}
$$

Since $W-\lim _{n \rightarrow \infty} v_{n}=\bar{v}, \quad \lim _{n \rightarrow \infty}\left(\int K\left[\bar{v}\left(s_{k}\right)-v_{n}\left(s_{k}\right)\right] d s_{k}\right)=0$, and since $\left|\int K\left(\bar{x}, s_{1}, \cdots, s_{i}\right)\left[\bar{v}\left(s_{k}\right)-v_{n}\left(s_{k}\right)\right] d s_{k}\right| \leqq 2 N(b-a) \cdot \max |K|$, we see that the sequence of Lebesgue integrable functions $\left(\int K\left(\bar{x}, s_{1}, \cdots, s_{i}\right)\right.$ $\left.\cdot\left[\bar{v}\left(s_{k}\right)-v_{n}\left(s_{k}\right)\right] d s_{k}\right)^{2}$ has a common bound and the limit function zero. By Lebesgue's convergence theorem we may conclude that

$$
\lim _{n \rightarrow \infty} \int \cdots \int\left(\int K\left[\bar{v}\left(s_{k}\right)-v_{n}\left(s_{k}\right)\right] d s_{k}\right)^{2} d s_{1} \cdots d s_{k-1} d s_{k+1} \cdots d s_{i}=0
$$

for $k=1,2, \cdots, i$, so that the proof of the full continuity of $Q(x, v)$ is now complete.

6. A special case. The deductions of $\$ 4$ are therefore applicable to the integral equation

$$
\begin{aligned}
& \phi(x)=\lambda\left[f(x)+\sum_{i=1}^{m} a_{i} \int \cdots \int K_{i}\left(x, s_{1}, \cdots, s_{i}\right)\right. \\
&\left.\cdot \phi\left(s_{1}\right) \cdots \phi\left(s_{i}\right) d s_{1} \cdots d s_{i}\right] .
\end{aligned}
$$


If we assume that $a_{m}=1, a_{i}=0$ for $i=1,2, \cdots, m-1$, that is, if we consider

$$
\begin{aligned}
\phi(x)=\lambda\left[f(x)+\int \cdots \int K_{m}\left(x, s_{1}, \cdots, s_{m}\right)\right. & \\
& \left.\cdot \phi\left(s_{1}\right) \cdots \phi\left(s_{m}\right) d s_{1} \cdots d s_{m}\right],
\end{aligned}
$$

we know that it has at least one solution, and that this solution may belong to a finite or an infinite eigenvalue. The homogeneous equation

$$
\phi(x)=\lambda \int \cdots \int K_{m}\left(x, s_{1}, \cdots, s_{m}\right) \phi\left(s_{1}\right) \cdots \phi\left(s_{m}\right) d s_{1} \cdots d s_{m},
$$

however, has always at least one finite eigenvalue. In this case namely (see (6))

$$
J\left(v_{n}\right)=2 e_{m} \int G_{m}\left(x, v_{n}\right) v_{n}(x) d x
$$

so that

$$
d_{n}=\frac{2}{m+1} \int G_{m}\left(x, \phi_{n}\right) \phi_{n}(x) d x=\frac{2}{m+1} \cdot \frac{N^{2}}{\lambda_{n}}
$$

or

$$
\lambda_{n} \cdot d_{n}=(2 /(m+1)) N^{2} .
$$

But since the functional

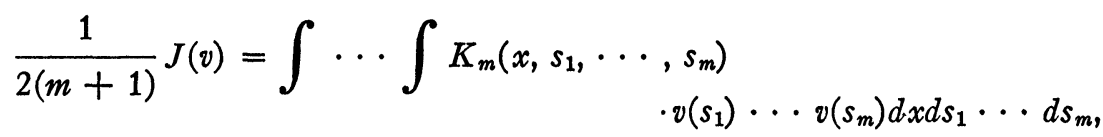

$K_{m} \not \equiv 0, v \in \bar{H}^{2}$, certainly has a minimum $\bar{d}$ differing from zero,

$$
\bar{\lambda}=\lim _{\bar{n} \rightarrow \infty} \lambda_{n}=\frac{2 N^{2}}{\bar{d}(m+1)}
$$

is finite.

Carleton College 